\title{
Mutational and hyperexpression-induced disruption of bipolar budding in yeast
}

\author{
Toby Freedman, ${ }^{1,2} \dagger$ Alexandra Porter ${ }^{2}$ and Brian Haarer ${ }^{2,3}$ \\ Author for correspondence: Brian Haarer. Tel: +1 315464 8757. Fax: +1 3154648750. \\ e-mail: haarerb@mail.upstate.edu
}

\footnotetext{
1 Department of Biochemistry and Molecular Biology, Harvard University, Cambridge, MA 02138, USA

2 Section of Molecular Cell and Developmental Biology, The University of Texas, Austin, TX 78712, USA

3 Department of Biochemistry and Molecular Biology, SUNY Upstate Medical University, $750 \mathrm{E}$. Adams St, Syracuse, NY 13210, USA
}

\begin{abstract}
Analysis of bud-site selection in the yeast Saccharomyces cerevisiae has helped to identify many genes that are generally important for eukaryotic cell polarization. Colony morphology screens were used to identify factors relevant to the process of bipolar budding in yeast. Mutants defective in bipolar budding were identified by virtue of their inability to grow as pseudohyphae in a haploid bud3 background. A mutant allele of the MYO2 gene, encoding a class-V unconventional myosin was identified that perturbs bipolar budding without affecting axial budding and without grossly affecting the role of Myo2p in secretion and maintenance of the actin cytoskeleton. Several genes were also identified whose products, when overexpressed, are capable of disrupting bipolar budding. Among these are the actin-monomer-binding protein profilin and the Aip3p/Bud6p-interacting protein Atc1p. The results strongly support involvement of the actin cytoskeleton in the establishment of bipolar budding and in the maintenance of pseudohyphal growth.
\end{abstract}

Keywords: Polarity, actin, profilin, MYO2, myosin

\section{INTRODUCTION}

Cellular reproduction and organismal development are dependent upon the ability of cells to determine an axis of polarity. The budding yeast Saccharomyces cerevisiae displays distinct patterns of polarity with respect to its choices in bud position on the mother cell, organization of the newly emerging bud and in directing alternative developmental patterns, including pseudohyphal growth, mating, and sporulation (Chant, 1996; Gimeno et al., 1992; Roemer et al., 1996a). The choice of a site for bud emergence is strongly dependent upon the genetic composition of the mating-type locus, with those cells that are homozygous for mating type displaying axial budding (proximal pole with respect to the motherbud junction) and cells heterozygous for mating type displaying bipolar budding (predominantly distal pole budding in the daughter cell and proximal or distal pole budding in the mother cell). The mechanisms regulating axial versus bipolar budding show distinct differences, such that independent mechanisms have been proposed for these bud-site selection pathways (Chant, 1996; Chant \& Pringle, 1995; Zahner et al., 1996).

† Present Address: SLIL Biomedical Corp., Menlo Park, CA 94025, USA. Abbreviations: $\mathrm{PIP}_{2}$, phosphatidylinositol 4,5-bisphosphate
Budding patterns are also influenced by nutrient availability in strains that are competent to grow as pseudohyphae. Upon appropriate nutrient deprivation, such strains undergo a specialized form of bipolar budding in which daughter cells bud distal and mother cells bud proximal to the mother-bud junction (Gimeno et al., 1992). Further, mother and daughter cells become more elongated and have synchronous cell-division cycles, rather than displaying the normally observed lag in daughter cell division (Kron et al., 1994). Such pseudohyphal growth is thought to allow cells to forage for limiting nutrient sources, and requires the ability to grow in a bipolar pattern. Pseudohyphal growth also requires the actin cytoskeleton to properly organize in a polarized fashion (Cali et al., 1998). Indeed, mutations that perturb actin function can disrupt both bipolar budding and pseudohyphal growth, while having little or no adverse effect on axial budding (Haarer et al., 1996; Yang et al., 1997).

Due to the strong dependence of pseudohyphal growth and bipolar budding on the actin cytoskeleton, we have sought to learn more about these relationships, and thus about regulation of the actin cytoskeleton, by identifying conditions that perturb normal bipolar budding. We have screened for mutants that are defective in bipolar budding and pseudohyphal growth, and have also randomized budding through gene overexpression. We 
have found that mild mutation of a class- $\mathrm{V}$ myosin, encoded by MYO2, eliminates bipolar budding. We also found that overproduction of the actin-regulatory protein profilin perturbs bipolar budding, and that profilin's ability to interact with actin is important for this effect. These and other results confirm the essential nature of the actin cytoskeleton for normal polarization in $S$. cerevisiae.

\section{METHODS}

Yeast strains, plasmids and growth conditions. Saccharomyces cerevisiae strains used in this study are listed in Table 1. Cells were generally grown on rich medium (YEPD) or synthetic defined medium (SD) containing appropriate nutritional supplements to maintain plasmid selection (Sherman et al., 1986). For induction of pseudohyphal growth, strains were grown on the pseudohyphal growth medium SLAD (Gimeno et al., 1992). Unless otherwise indicated, growth of yeast strains was at room temperature $\left(24^{\circ} \mathrm{C}\right)$ or $30^{\circ} \mathrm{C}$. Plasmid YCp(ACT1) was provided by D. Drubin and $\mathrm{K}$. Wertman (Dept. Molecular and Cellular Biology, University of California, Berkeley, USA); YCpMYO2 (Lillie \& Brown, 1994) was provided by S. Lillie (Dept. Cell and Molecular Biology, University of Michigan, Ann Arbor, USA). Plasmid YEp352(MYO4) has been described previously (Haarer et al., 1994).

DNA manipulations and yeast genetic techniques. Standard procedures were used for recombinant DNA manipulation and Escherichia coli transformation (Sambrook et al., 1989). Yeast transformation was by the lithium acetate procedure (Ma et al., 1987).

Mutant screen and assessment of budding patterns. Haploid bud3 strains TFY147 and TFY149, which bud bipolarly and are capable of pseudohyphal growth, were mutagenized with EMS and plated on YEPD at room temperature. Colonies were replica plated on SLAD media and examined with a dissecting microscope after $3 \mathrm{~d}$. Potential pseudohyphaldefective mutants were restreaked on YEPD, then replica plated on SLAD media to confirm the mutant phenotype. Screening of $\sim 40000$ colonies yielded 146 pseudohyphaldefective mutants, of which 49 were defective in bipolar budding. The bud-site selection pattern was assayed by staining exponential-phase cells from liquid YEPD cultures with the chitin-specific dye calcofluor white (Pringle et al., 1989). Bud-site selection was scored in cells having more than one calcofluor white-stained bud scar. Cells having scars at both poles, but not in the middle, were scored as bipolar, while cells having at least one scar not at either pole were scored as random. Six random-budding mutants with the strongest phenotypes were chosen for further analysis. Of these, genetic crosses showed that five were the result of single-locus mutations. Attempts to clone the responsible genes by complementation of the pseudohyphal-defective phenotype proved unproductive, as the frequency of reversion (presumably through second-site mutations) was quite high. Therefore, the randomizing mutations were introduced into the 'rough colony' background of strain TFY209 (see below) and candidate genes were obtained by screening for a smooth to rough colony transition upon transformation with yeast genomic DNA libraries.

Complementation tests of bipolar-defective mutants. Mutations that disrupted bipolar budding were tested against each other and against mutations in known genes in the following complementation assay. Strains of opposite mating type, of which one strain was ura3 HIS4 and the other was URA3 his4, were mixed together on SLAD plates lacking uracil and histidine. The resulting colonies, which should arise from ura3/URA3 HIS4/his4 diploids, were then examined for pseudohyphal growth. The absence of pseudohyphal growth was considered to be non-complementation and the corresponding mutations were placed in the same complementation group. Conversely, demonstration of pseudohyphal growth resulted in placement of the mutations in separate complementation groups. Most pairwise complementation tests were repeated by using strains of the opposite mating type for the given mutations. In three instances, one cross failed to complement, while the reciprocal cross yielded a positive complementation result. In these cases, the mutations were considered to represent separate complementation groups. Mutations that fell into unknown complementation groups were tested for bipolar specificity by generating the corresponding BUD3 haploid strains.

Overproduction screen. A multicopy genomic yeast DNA library in plasmid YEp13 was transformed into the bipolarbudding bud3 strain TFY209, which exhibits a rough colony morphology. While not well characterized, this rough colony phenotype arises from cells that are budding in a bipolar pattern. Axial or random budding results in a smooth colony morphology. In this screen, 323 of $\sim 14000$ transformed colonies exhibited a reduction of the rough colony morphology. Of these transformants, 12 budded in an axial pattern (due to acquisition of BUD3-containing plasmids) and 30 showed random budding. The remaining 281 transformants exhibited normal bipolar budding and were not examined further. Plasmids were isolated from each bipolar-defective transformant and retested. Subcloning and sequence analysis

Table 1. S. cerevisiae strains used in this study

\begin{tabular}{|c|c|c|}
\hline Strain & Relevant genotype & Source \\
\hline TFY147 & MATa bud3:: TRP1 ura3 & This study \\
\hline TFY148 & MAT $\alpha$ bud3:: TRP1 his 4 ura 3 & This study \\
\hline TFY149 & MAT $\alpha$ bud3:: TRP1 his4 ura3 + plasmid YCp50 & This study \\
\hline TFY209 & MAT $\alpha$ bud3A::URA3 ura3 leu 2 trp1 & This study \\
\hline TFY211 & MATa bud3A::URA3 ura3 leu 2 trp1 & This study \\
\hline TFY250 & MATa/MATa myo2-117/myo2-117 & This study \\
\hline TFY 648 & MATa/MATa leu $2 /$ leu $2 \operatorname{trp} 1 / \operatorname{trp} 1$ ura $3 /$ ura 3 can $1 /$ can 1 & J. Chant* \\
\hline
\end{tabular}

*Dept. Molecular and Cellular Biology, Harvard University, Cambridge, MA, USA. 
revealed the genes responsible for perturbing bipolar budding, as summarized in Table 3.

\section{RESULTS}

\section{Mutations in MYO2 disrupt bipolar but not axial budding}

Under specific nitrogen-starvation conditions, certain $S$. cerevisiae strains are induced to switch from the yeast form to the filament-like pseudohyphal form (Gimeno et al., 1992). Cells become highly elongated, remain loosely attached to each other and appear as long chains. Bipolar budding is one requirement for pseudohyphal growth (Gimeno et al., 1992), as wild-type haploid a or $\alpha$ cells, which bud axially, are not competent to grow pseudohyphally. Wild-type diploid a/ $\alpha$ cells, which bud in a bipolar pattern, grow as pseudohyphae when cultured on appropriate nitrogen-limited media. However, a $/ \alpha$ cells that are unable to bud in a bipolar fashion (e.g. bud1, bud2 and bud5 mutants) are incapable of
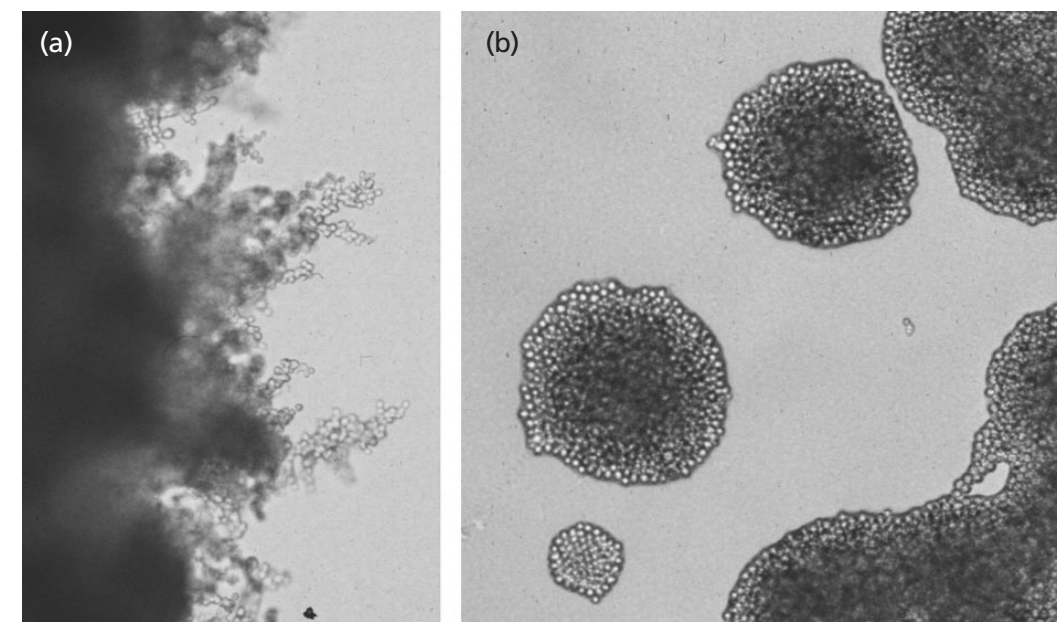

Fig. 1. Bipolar-budding defective mutants fail to undergo pseudohyphal growth. Shown are (a) strain TFY149 and (b) representative random-budding mutant TFY55; growth was on SLAD medium at $25^{\circ} \mathrm{C}$ for $5 \mathrm{~d}$.
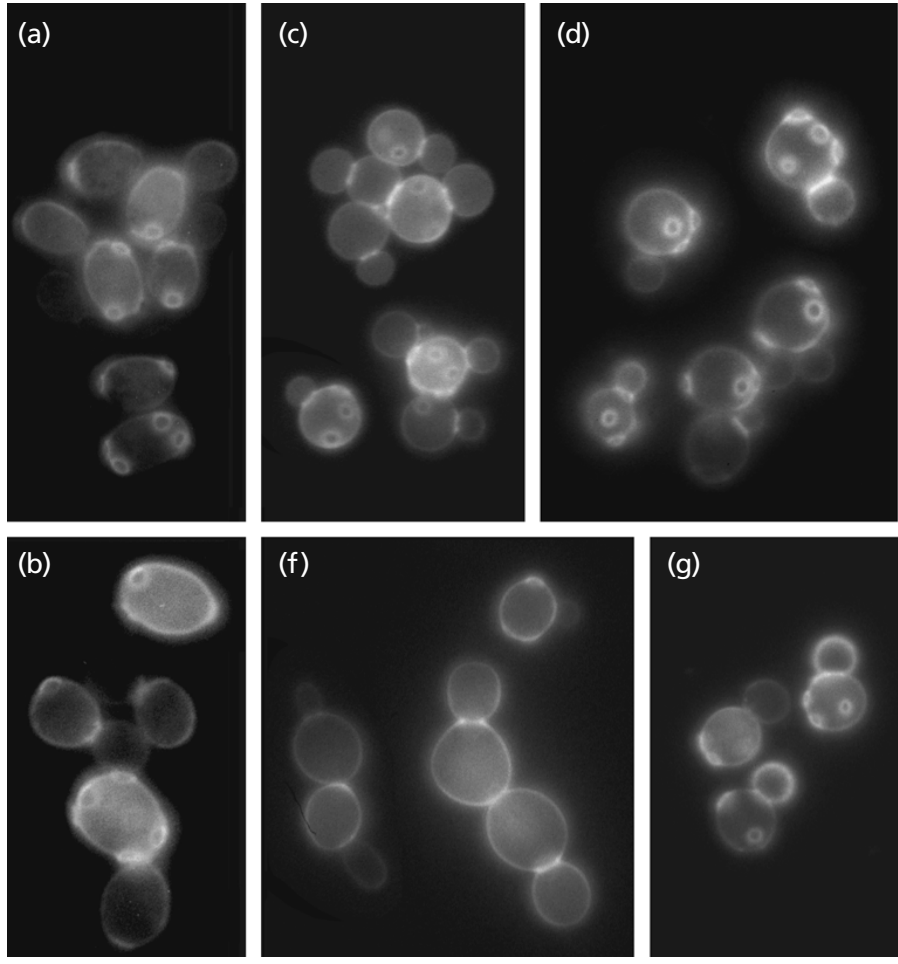

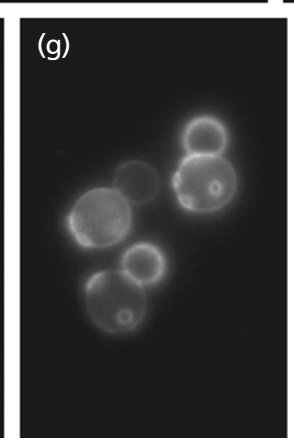

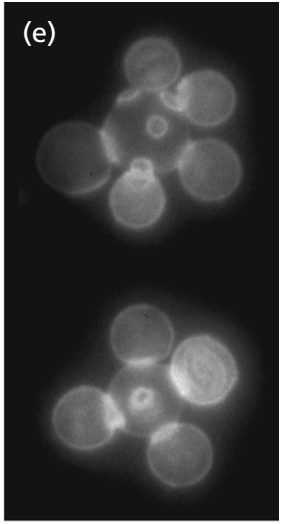

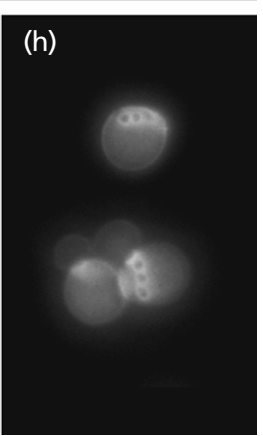

Fig. 2. Bud-site selection in myo2 mutant strains. Shown are calcofluor white-stained cells from control strains TFY147 (a) and TFY149 (b), and from strains of genotype myo2-117 bud3 (c); myo2-117/myo2-117 (d); myo2-117 bud3 bud4, grown at $37^{\circ} \mathrm{C}(\mathrm{e})$; myo2-117 bud3, containing plasmid YCpMYO2 (f); myo2-66 bud3 (g); and myo2-66 (h). Unless otherwise indicated, growth was at $25^{\circ} \mathrm{C}$ in YEPD. 

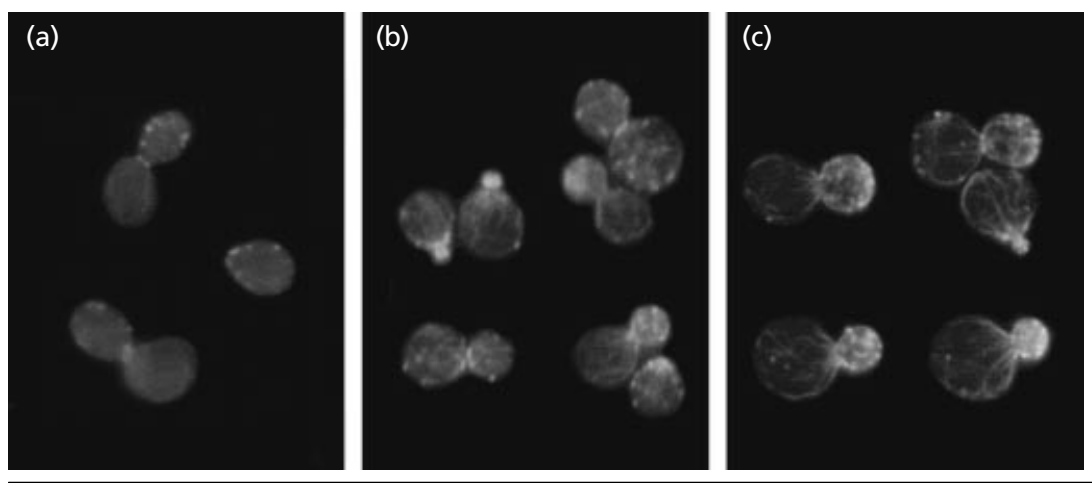

Fig. 3. Actin distribution in myo2-117 cells. Control strain TFY147 (a), myo2-117 bud3 strain TFY14 (b), and myo2-117/myo2-117 bud3/bud3 strain TFY250 (c) stained with rhodamine-conjugated phalloidin.

Table 2. Partial suppression of the myo2-117/myo2-117 bipolar budding defect by actin overexpression

Strain TFY250 was used in all cases. The following criteria were used to assess budding patterns of cells having two or more bud scars: unipolar, all scars at one pole of the cell only; bipolar, scars at both poles of the cell, but not in the middle; random, at least one scar not at either pole. Values are shown as percentages and represent the mean of two experiments; $n=150-200$ cells for each transformant per replicate.

\begin{tabular}{|lccc|}
\hline Plasmid* & \multicolumn{3}{c|}{ Budding Pattern } \\
\cline { 2 - 4 } & Random & Bipolar & Unipolar \\
\hline YEp352 & 70 & 20 & 10 \\
YCp(ACT1) & 55 & 37 & 8 \\
YEp(MYO4) & 70 & 19 & 11 \\
\hline
\end{tabular}

*YEp352, control vector; YCp(ACT1), low-copy-number plasmid carrying the yeast actin gene; YEp352(MYO4), highcopy-number plasmid carrying the MYO2-related MYO4 gene.

growing as pseudohyphae (Gimeno et al., 1992). Conversely, haploid bud3 or bud4 mutants, which bud in a bipolar pattern, are capable of pseudohyphal growth (Sanders \& Herskowitz, 1996) (see Fig. 1a).

Zhaner et al. (1996) have previously used a bud3 strain to microscopically screen for mutants that are defective for bipolar budding. Using a similar haploid bud3 strain, we carried out a screen for mutants that are unable to grow pseudohyphally. Of 146 such mutants (see Fig. 1b), 49 were found to display random budding patterns. The random-budding mutations were tested for complementation (see Methods) of each other and of known bipolar-defective mutations in BUD1/RSR1, BUD2, BUD5, SPA2, RVS161, RVS167, SUR4 and SUR5 (Bauer et al., 1993; Bender \& Pringle, 1989; Chant et al., 1991, 1995; Chant \& Herskowitz, 1991; Crouzet et al., 1991; Durrens et al., 1995; Park et al., 1993; Powers et al., 1991; Revardel et al., 1995; Snyder, 1989). The complementation data suggested that we obtained mutations in BUD1 (one isolate), BUD2 (nine isolates), BUD5 (five isolates) and SPA2 (one isolate). In addition, one mutation failed to complement bud1 and bud2 mutations, and thus is likely to correspond to one of these genes. Oddly enough, the remaining 23 mutations fall into separate complementation groups (nine mutations convey a mating defect and were not included in the complementation test). Thus, there is either a high incidence of interallelic complementation among these mutations, or there are a great number of mutations that will generate bipolar budding defects (at least in this genetic background; see Discussion).

Six mutant strains were chosen from this collection as having the greatest randomizing effect on bipolar budding. A complementing plasmid clone was identified for one of these strains and was found to contain the MYO2 gene, which encodes a type- $\mathrm{V}$ myosin previously shown to be essential for polarized secretion (Govindan et al., 1995; Johnston et al., 1991; Lillie \& Brown, 1994). Subsequent crosses to a myo2-66 mutant confirmed that the random-budding mutation is a novel allele of $M Y O 2$ and will hereafter be referred to as myo2-117. Complementing genes for the remaining mutations have not yet been identified.

The myo2-117 allele causes normally bipolar-budding cells to bud in a random fashion, but does not affect axial budding. This is true both for haploid myo2-117 bud3 cells (Fig. 2c) and diploid myo2-117/myo2-117 cells (Fig. 2d). While the myo2-117 mutation does not result in the severe temperature sensitivity caused by the myo2-66 mutation, myo2-117 cells do display a partial cell-separation defect upon prolonged incubation at $37^{\circ} \mathrm{C}$ (Fig. 2e). Zymolyase treatment of fixed myo2-117 cells that were grown at $37^{\circ} \mathrm{C}$ results in dissociation of daughter cells, suggesting that the defect is in cell-wall separation, rather than in cytokinesis (data not shown). The defects associated with the myo2-117 allele are recessive and are efficiently rescued by plasmid-borne MYO2 (Fig. 2f). Our observations of myo2-117-containing strains caused us to re-examine the previously described myo2-66 allele (Johnston et al., 1991; Lillie \& Brown, 1994) for similar effects. At permissive temperatures, haploid myo2-66 bud3 strains bud in a random pattern (Fig. 2g), while corresponding myo2-66 strains bud in the normal axial pattern (Fig. $2 \mathrm{~h}$ ). As with myo2-117 strains, homozygous myo2-66 diploid strains bud randomly (data not shown). Interestingly, a myo2- 

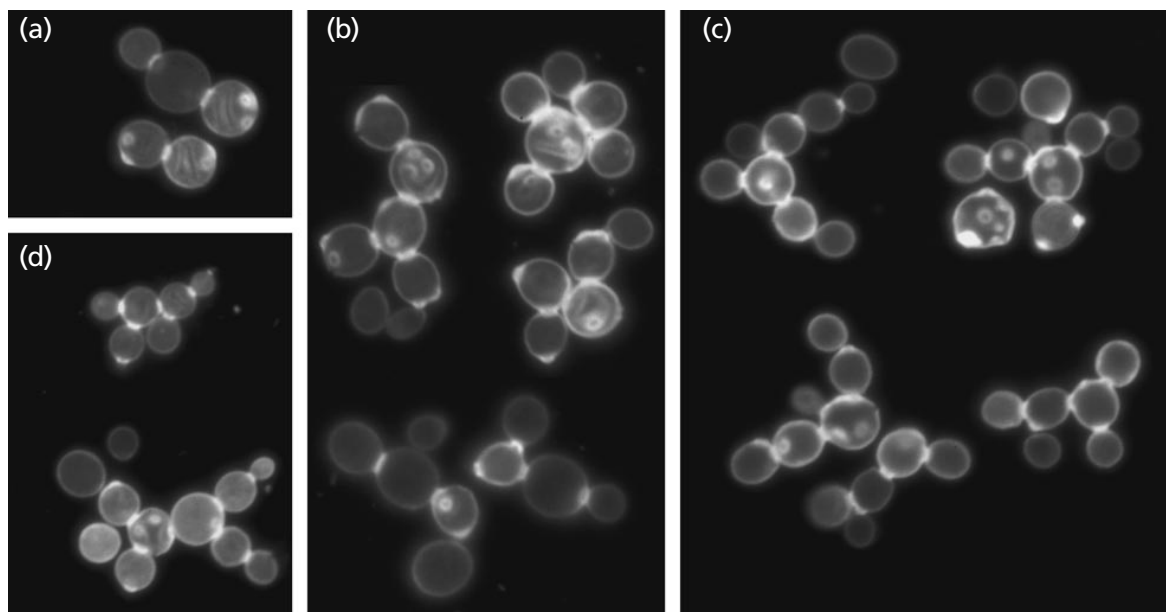

Fig. 4. Randomization of bipolar budding by gene overexpression. Calcofluor white-stained cells of strain TFY209 carrying control plasmid YEp351 (a), or library clones 414 (BUD10) (b); 422 (PFY1) (c); or 75 (ATC1) (d).
$117 /$ myo2-66 diploid is partially restored for bipolar budding, suggesting partial interallelic complementation between these mutant myo2 genes.

To determine if the random budding of myo 2 strains is the result of an inability to properly localize potential bud-site determinants, we examined the distribution of several proteins known to be important for normal polarized budding. Rhodamine-phalloidin staining showed that actin is properly localized in myo2-117 bud3 haploid and myo2-117/myo2-117 diploid strains (Fig. 3). Use of green fluorescent protein fusions further indicated that septins (Cdc3p) and the axial budding determinant Bud10p/Axl2p, which colocalize to the mother-bud neck, are positioned normally in myo2-117 mutant cells (data not shown). Despite the normal appearance of the actin cytoskeleton, we did find that mild overexpression of yeast actin partially suppresses the bipolar budding defect of a myo2-117/myo2-117 strain (Table 2), while overexpression of another type $\mathrm{V}$ myosin (Myo4p) has no effect.

Lillie \& Brown (1992) have shown that increased expression of SMY1, which encodes a kinesin-related protein, can partially rescue the temperature sensitivity of strains carrying the myo2-66 mutation. Expression of SMY1 from a high-copy-number plasmid fails to suppress the bipolar budding defect of a myo2-117 bud3 strain. Moreover, a smy1/smy1 homozygous diploid buds in a normal bipolar pattern, and a haploid smy1 myo2-117 strain buds in a normal axial pattern (data not shown). These results suggest that Smy1p activity is more relevant to the essential role of Myo2p and may have little to do with the participation of Myo2p in organizing budding polarity.

\section{Multicopy randomization of bipolar budding}

To complement our mutational studies, we also carried out a screen to identify genes that, when overexpressed, disrupt bipolar budding in a bud3 haploid strain. In this screen, we utilized strain TFY209, which carries a bud3 deletion mutation and exhibits a rough colony mor- phology. This rough colony morphology is similar to pseudohyphal growth, in that it requires bipolar budding and can occur in a haploid bud3 or bud4 background. However, unlike pseudohyphal growth, the rough colony morphology occurs under non-starvation conditions and thus does not require specialized growth media. We transformed strain TFY209 with a high-copy yeast genomic library and screened for smooth colonies arising on selectable media. Candidate colonies were retested to confirm the smooth colony phenotype and cells were stained with calcofluor white to determine if the bipolar budding pattern was disrupted (representative transformants are shown in Fig. 4). Of 323 smooth colonies, 30 budded in a random pattern and 12 budded in an axial pattern. Plasmid clones were isolated from bipolar-defective transformants and were retested for their ability to promote the smooth-colony phenotype. Clones from the 12 axial budding transformants were found to contain the BUD3 gene; clones from the random budding transformants are discussed below.

Sequence was obtained from the ends of yeast DNA inserts of plasmid clones and compared to the yeast genome database to identify the genes contained on each plasmid (Table 3 ). In many cases, further subcloning unambiguously identified individual genes that disrupt bipolar budding in the bud3 strain. To further examine the nature of this perturbation, we tested the plasmids in other genetic backgrounds, including bud3/bud3 and $\mathrm{Bud}^{+}$diploids (Table 3). Our results indicate that most of the clones disrupt bipolar budding only when BUD3 function is also perturbed. However, we have not ruled out strain background effects as contributing to these results (see Discussion).

\section{Overproduction of profilin or Atc1p disrupts bipolar budding}

The quantitatively strongest inhibitor of bipolar budding that we obtained was PFY1 (see Fig. 4c), which is the sole profilin gene in yeast. Profilin is an actinmonomer-binding protein that is important for vegetative growth and essential for normal polarized 
Table 3. Multicopy randomization of bipolar budding

\begin{tabular}{|c|c|c|c|c|c|}
\hline \multirow[t]{2}{*}{ Clone } & \multirow[t]{2}{*}{ Relevant gene $(s)[\mathrm{ORF}(\mathrm{s})]$ on clone* } & \multirow[t]{2}{*}{$\begin{array}{l}\text { Independent } \\
\text { Isolates }\end{array}$} & \multicolumn{3}{|c|}{$\begin{array}{c}\text { Random budding in selected } \\
\text { yeast strains }(\%)\end{array}$} \\
\hline & & & $\begin{array}{c}M A T \alpha \\
b u d 3 \Delta\end{array}$ & $\begin{array}{c}M A T \mathrm{~T} / M A T \alpha \\
\operatorname{bud} 3 \Delta / \mathrm{bud} 3 \Delta\end{array}$ & $\begin{array}{l}M A T a / M A T \alpha \\
B U D 3 / B U D 3 \dagger\end{array}$ \\
\hline 422 & PFY1 (YOR122c) & 1 & 57 & 67 & BP \\
\hline 170,414 & BUD10 (YIL140w) & 5 & 36 & 38 & $\mathrm{~A} / \mathrm{BP}$ \\
\hline 75 & ATC1 (YDR184c) & 2 & 41 & 62 & $\mathrm{BP}$ \\
\hline 118 & PDE2 (YOR360c) & 3 & 53 & 33 & ND \\
\hline 73 & MLP1 (YKR095w) & 1 & 26 & 34 & 17 \\
\hline 241 & {$[$ SAP4 (YGL229c)]‡ } & 1 & 66 & 21 & $\mathrm{BP}$ \\
\hline 106 & MEC3 (YLR288c) & 1 & 37 & 11 & BP \\
\hline 78 & $\begin{array}{l}\text { [YPL067c], YPL068c, BTS1 (YPL069c), YPL070w, } \\
{[\text { YPL071c] }}\end{array}$ & 1 & 35 & 23 & 7 \\
\hline 114 & YDR333c & 1 & 43 & 32 & 2 \\
\hline 420 & [HYP2 (YEL034w)], YEL033w & 1 & 39 & 31 & $\mathrm{BP}$ \\
\hline 87 & {$[Y M R 247 c] \mathbb{S}$} & 1 & 29 & 16 & ВP \\
\hline 143 & {$[$ YOR011w]\| } & 1 & 60 & 15 & 1 \\
\hline 418 & [SRM1 (YGL097w)], YGL096w, delta element & 1 & 20 & 31 & вр \\
\hline
\end{tabular}

*In cases where the effect has not been narrowed down to a single gene, all candidate genes are indicated. If previously characterized, the gene name is given first, with the yeast database ORF designation in parentheses; square brackets indicate an incomplete gene/ORF on clone.

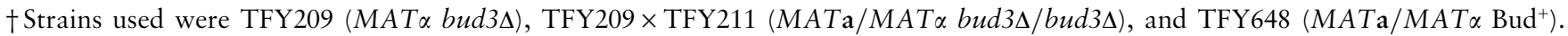
For each data point, $n \geqslant 100$ cells; BP designates little or no departure from normal bipolar budding; A indicates an axial appearance of some cells in a population of cells that are overexpressing Bud10p; ND, not determined.

$\ddagger$ Predicted to express the first 410 of 818 codons of SAP4.

SPredicted to express the first 1309 of 1562 codons of YMR247c.

|| Predicted to express the first 1148 of 1394 codons of YOR011w.

budding in yeast (Haarer et al., 1990; Sohn \& Goldschmidt-Clermont, 1994). Deletion of PFY1 results in randomized budding in haploids (Haarer et al., 1990), while various PFY1 point mutations result in randomized budding in diploids, but do not affect the normal axial budding of haploid strains (Haarer et al., 1996; T. Freedman and B. Haarer, unpublished data). These results suggest that bipolar budding requires stringent controls on the levels of profilin expressed in the cell. This is in contrast to the relaxed cellular requirements regarding profilin quantity and function necessary to permit relatively normal vegetative growth (Haarer et al., 1993, 1996; Magdolen et al., 1993).

Profilin is able to interact with various substrates, including actin, phosphatidylinositol 4,5-bisphosphate $\left(\mathrm{PIP}_{2}\right)$ (Sohn \& Goldschmidt-Clermont, 1994) and the proteins Bnilp and Bnr1p, which are related to each other in structure and have been implicated in actin and cell polarization (Evangelista et al., 1997; Imamura et al., 1997). Interaction of profilin with Bni1p and Bnr1p is through multiple proline residues, and requires profilin's characterized polyproline-binding activity (Evangelista et al., 1997; see also Haarer et al., 1993). To better understand profilin's perturbing effects on bipolar bud-site selection, we assessed the budding randomization effects of various mutant profilins that
Table 4. Effects of profilin mutation on multi-copy randomization of bud-site selection

Strain TFY148 was used in all cases. See Table 2 legend for budding pattern criteria. For each sample, $n=250-300$. Values shown are percentages.

\begin{tabular}{|lccc|}
\hline Plasmid* & \multicolumn{3}{c|}{ Budding Pattern } \\
\cline { 2 - 4 } & Random & Bipolar & Unipolar \\
\hline YEp352 & 20 & 52 & 28 \\
PFY1 & 44 & 51 & 5 \\
pfy1-111 & 26 & 62 & 12 \\
pfy1-112 & 51 & 30 & 19 \\
pfy1-115 & 28 & 47 & 25 \\
pfy1- $\triangle C 3$ & 49 & 40 & 11 \\
\hline
\end{tabular}

*YEp352, control vector; Wild-type (PFY1) or mutant ( $p f y 1-111$, $p f y 1-112, p f y 1-115, p f y 1 \Delta C 3)$ profilin alleles were expressed from high-copy-number URA3-based plasmids.

are defective in one or more of these binding activities (Table 4). The $p f y 1-111$ and $p f y 1-115$ alleles produce profilins that exhibit greatly reduced interaction with actin, while $p f y 1-112$ profilin is only moderately affected

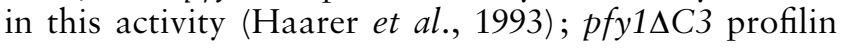


(-3PFY in Haarer et al., 1993) is largely defective in binding polyproline and fails to interact with Bni1p (Evangelista et al., 1997; S. Palmieri and B. Haarer, unpublished data). Based on these data (Table 4), the ability of profilin to randomize budding correlates with its actin-binding activity and is independent of its ability to interact with proline-rich substrates. While we cannot rule out an interaction with $\mathrm{PIP}_{2}$ as contributing to this effect, it is unlikely to be the sole activity responsible for bud-site randomization, as $p f y 1-111$ profilin is defective in $\mathrm{PIP}_{2}$ binding, while $p f y 1-115$ profilin is not (Haarer et al., 1993).

Overproduction of another gene implicated in actin regulation also results in a partial disruption of bipolar budding. The product of the ATC1 gene was identified via its interaction with Bud6p/Aip3p in the two-hybrid system (D. Amberg, personal communication; and Saccharomyces Genome Database, http://genomewww.stanford.edu/Saccharomyces/). Bud6p/Aip3p is an actin-binding protein that is essential for normal bipolar budding (Amberg et al., 1995, 1997; Zahner et al., 1996). Evangelista et al. (1997) have shown that Bud6p/Aip3p binds to the formin Bnilp and is probably recruited to sites of Cdc $42 p$ or Rho1p activation through this interaction. Thus, overproduction of Atc1p may exert its effects on bipolar budding by perturbing the normal localization or binding activity of Bud6p/Aip3p. Interestingly, this putative signalling complex also includes profilin, which is recruited by Bnilp. As indicated above, profilin overproduction also disrupts bipolar budding, perhaps through perturbation of this signalling complex or simply by decreasing the pool of actin monomers available to contribute to actin filament formation or binding to Bud6p/Aip3p. We have recently found that overproduction of the Bnilp homologue Bnr1p can largely overcome the budding defects caused by profilin overproduction (T. Freedman and B. Haarer, unpublished). This suppression may occur via enhanced signalling through Bnr1p, or may simply be due to sequestration of excess profilin.

\section{Overexpression of BUD10/AXL2 and BUD1/RSR1 disrupt bipolar budding}

The most frequently obtained multicopy disrupter of bipolar budding in our screen was BUD10/AXL2 (see Fig. 4b), a gene previously identified via its requirement for axial budding (Halme et al., 1996; Roemer et al., 1996b). This is particularly interesting, as mutation of BUD10/AXL2 results in bipolar budding in strains that would otherwise bud in an axial pattern. While Bud10p/ Axl2p overproduction tends to randomize bud-site selection, a low percentage of cells bud in an axial pattern (Table 3). Further examination of this phenomenon will be presented in a separate study (T. Freedman and J. Chant, unpublished).

A plasmid clone containing the gene for the Ras-related GTPase Bud1p/Rsr1p was also identified as a disrupter of bipolar budding, although it is much less effective at perturbing bud-site selection and only had a significant effect in the bud3 haploid background (data not shown). $B U D 1 / R S R 1$ is essential for maintaining proper axial and bipolar budding polarity (Bender \& Pringle, 1989; Chant \& Herskowitz, 1991), as are its GTPaseactivating protein and guanine nucleotide exchange factor, Bud2p and Bud5p, respectively (Bender, 1993; Chant et al., 1991; Park et al., 1993; Powers et al., 1991). Further, an activated allele of the Bud1/Rsr1 GTPase fails to rescue the bud-site selection defects of a bud1/rsr1 deletion strain (Ruggieri et al., 1992). Thus, it is not surprising that extra copies of the wild-type BUD1/RSR1 gene disrupt bipolar budding in our strain.

\section{Additional multicopy disrupters of bipolar budding}

Several other multicopy disrupters of bipolar budding were obtained in this study, as indicated in Table 3. Space constraints preclude extensive discussion regarding the possible effects these genes may have on polarity determinants; however, of particular note to the issue of bipolar budding and pseudohyphal growth is the gene PDE2. PDE2 encodes a high-affinity phosphodiesterase that is responsible, along with the product of the PDE1 gene, for decreasing cAMP levels. This is crucial for reversing the effects of the Ras-stimulated adenylate cyclase pathway in yeast, which is responsible for growth stimulation in response to nutrient availability. Ward et al. (1995) have demonstrated that PDE2 inhibits pseudohyphal growth. Further, Ras is necessary for pseudohyphal growth, and activated RAS2 alleles can drive pseudohyphal growth under non-starvation conditions (Gimeno et al., 1992). The G-protein alpha subunit encoded by GPA2 also stimulates pseudohyphal growth through elevation of cAMP levels (Lorenz \& Heitman, 1997). Given these data, it is not surprising that PDE2 overexpression suppresses the pseudohyphal response by decreasing cAMP levels. Beyond these specific effects on the pseudohyphal pathway, our results demonstrate that overexpression of PDE2 is also capable of disrupting bipolar budding, presumably in the absence of upstream activation of the pseudohyphal pathway (i.e. without GPA2 or RAS2 activation).

\section{DISCUSSION}

\section{Use of colony morphology as an indicator of bipolar budding and cytoskeletal function}

In this study, we have used the bipolar budding requirement for pseudohyphal growth and colony morphology to screen for factors that, when mutated or overproduced, can perturb this aspect of cell polarization. While the bud3 strain background used for these studies is similar to that utilized by Zahner et al. (1996), the use of colony morphology as an indicator of budding pattern, rather than direct microscopic examination, has greatly streamlined the process of identifying factors that affect bipolar budding. Furthermore, the preponderance of bipolar budding defects arising from mutations in genes that relate to actin cytoskeletal function (see Ayscough et al., 1997; Haarer et al., 1996; Palmieri \& Haarer, 1998; Yang et al., 1997, and 
references therein) suggested that such an approach could identify additional actin regulatory genes and may help to explain the involvement of such factors in the maintenance of bipolar budding.

It is apparent that our screens have identified factors that are involved in one or more of the following morphogenetic processes: bipolar budding, pseudohyphal determination, pseudohyphal growth or actin polarization. Indeed, it may not be possible to completely separate these functions, as bipolar budding and pseudohyphal growth require normal actin polarization (Cali et al., 1998; Mosch \& Fink, 1997; Yang et al., 1997), pseudohyphal growth generally requires unipolar budding, which is likely to be a specialized form of bipolar budding (Gimeno et al., 1992; Kron et al., 1994; Zahner et al., 1996) and bipolar budding may require some of the same activators that are necessary for pseudohyphal determination (Mosch \& Fink, 1997). Put more simply, the induction of pseudohyphal growth by nutritional signals requires the ability to bud bipolarly, which in turn requires normal actin polarization and regulation.

\section{Contribution of strain background to bipolar budding screens}

In our screen for multi-copy disrupters of bipolar budding, we noted that many of the clones identified had their most significant effect in bud3 mutant strains, most notably in the case of diploid strains. At present, we do not know if this effect is specific to bud3 or if there are subtle genetic differences between these strains that are contributing to the observed results. If the effect is indeed bud3-specific, this would imply a potential, albeit minor, role for Bud3p in bipolar budding. Attenuation of this function apparently makes yeast cells especially susceptible to additional perturbations in bipolar budding, thus enhancing their utility in such screens.

\section{Involvement of Myo2p in bud-site selection}

The relative lack of morphological abnormalities in strains expressing the myo2-117 allele suggests that the mutant myosin is able to provide the bulk of its normal cellular functions, particularly those relevant to secretion and cytoskeletal organization (Govindan et al., 1995; Johnston et al., 1991). Therefore, its inability to support bipolar growth suggests that the mutant protein is lacking in some non-essential function, presumably involving either an inability to interact with a protein that normally relies on Myo2p for its proper cellular distribution or an inability to properly position itself in the cell to deliver bipolar determinants. Catlett \& Weisman (1998) have identified an allele of MYO2 that is defective in vacuolar inheritance, bipolar bud-site selection and normal localization of the mutant $\mathrm{Myo} 2$ protein, but that is not grossly defective in bud growth or actin polarization. Thus, the role of Myo2p in budsite selection may require the efficient delivery of polarity determinants to the plasma membrane, perhaps via the secretory pathway. We suspect that Myo2p may help to position the proposed bipolar determinant(s) at presumptive budding sites, and that the observed effects are due to reduced efficiency of binding (directly or indirectly) to a polarity-determining cargo.

Myo2p has been strongly implicated in directing normal secretion in yeast (Govindan et al., 1995; Johnston et al., 1991; Lillie \& Brown, 1992, 1994), a process that is also dependent upon the actin cytoskeleton (Ayscough et al., 1997; Haarer et al., 1996; Novick \& Botstein, 1985). Surprisingly, Myo2p is able to polarize, albeit in a delayed fashion, independently of an intact actin cytoskeleton (Ayscough et al., 1997), which would suggest that it is able to directly respond to the global polarizing signal provided by Cdc42p. In contrast, Schott et al. (1999) found that Myo2p localization is dependent on actin. Consistent with this finding is the partial suppression of myo2-117-induced budding defects by overproduction of yeast actin (Table 2). Since perturbation of the actin cytoskeleton through mutation of various actin-accessory factors is likely to affect the timing and efficiency of Myo2p localization, it is possible that the general bipolar budding defects caused by such mutations (Yang et al., 1997) actually arise due to attenuation of Myo2p function and consequent mislocalization of a crucial polarity determinant. Thus, the situation may be analogous to the asymmetric delivery of the ASH1 mRNA and protein exclusively to daughter cells. This polarized delivery is strictly dependent upon the type- $\mathrm{V}$ myosin Myo4 $\mathrm{p}$ and is inhibited by factors that partially disrupt the actin cytoskeleton (Bobola et al., 1996; Jansen et al., 1996; Long et al., 1997; Takizawa et al., 1997).

The inability of SMY1 overproduction to suppress the bud-site selection defects of a myo2-117 strain argues against such defects resulting solely from reduced secretory efficiency; i.e. the proposed polarity determinant(s) may require direct interaction with Myo2p for proper positioning, such that simply increasing secretory efficiency would be insufficient to properly localize the determinant(s). It is also noteworthy that myo2-117/ myo2-66 heteroallelic diploids show an intermediate bipolar phenotype, whereas corresponding homoallelic diploids are incapable of supporting bipolar budding. Since the myo2-66 allele generates a change at the actinbinding face of the motor domain (Lillie \& Brown, 1994), it is possible that heterodimers of the two mutant myosins compensate by providing a single fully functional motor domain and a single wild-type binding site required for delivery of the hypothesized bipolar determinant(s).

\section{Actin-regulatory factors and bud-site selection}

Recent reports have stressed the importance of the actin cytoskeleton for various aspects of cell polarization, including bipolar bud-site selection and pseudohyphal growth (Ayscough et al., 1997; Cali et al., 1998; Haarer et al., 1996; Yang et al., 1997; Zahner et al., 1996). This may reflect a requirement of the actin cytoskeleton (and Myo2p) for properly placing or maintaining one or 
more positional cues that normally mark or determine bipolar budding sites. Potential candidates for such positional cues are the proteins Bud8p and Bud9p, defects of which result in loss of distal- or proximal-pole budding, respectively (Yang et al., 1997; Zahner et al., 1996), and Aip3p/Bud6p, an actin-interacting protein that is generally required for bipolar budding (Amberg et al., 1997; Zahner et al., 1996). Actin-mediated placement of these (or other) positional cues is likely to be indirect via effects on polarized secretion or myosinbased delivery of positional markers to polarized sites. Candidates for contributing to the delivery of positional cues include Sec3p and other components of the exocyst complex (Finger et al., 1998; Finger \& Novick, 1997; Haarer et al., 1996), in addition to Myo2p (see above). Defects in a subset of these secretory pathway components result in a randomization of bipolar budding, probably by reducing the efficiency with which positional cues achieve their polarized localization at or near the plasma membrane. Interestingly, normal polarization of Aip3p requires the secretory pathway and functional Myo2p (Jin \& Amberg, 2000).

As mentioned in the Results, profilin and Atc1p have been linked to Cdc42p and Rho protein-based signalling complexes through interactions with formins or forminassociated proteins (for a recent review, see Palmieri \& Haarer, 1998). It is likely that such signalling complexes serve to arrange the actin cytoskeleton into organized networks, which is critical for directing polarized secretion and for the distribution of polarity determinants. Presumably, the disruption of bipolar budding in strains that express various mutated profilins (Haarer et al., 1996; T. Freedman and B. Haarer, unpublished data) arises by significantly affecting the efficiency of these signalling complexes. Most likely, attenuated profilin function retards the rate of actin filament assembly at sites of Cdc42/Rho activation by reducing the rate of conversion of ADP-actin to ATP-actin or by failing to properly deliver bound actin monomers to the growing ends of actin filaments. While profilin may normally act in complexes that require its interaction with proline-rich proteins, its ability to disrupt bipolar budding upon overproduction probably rests in its ability to sequester actin monomers (Table 4). Thus, although profilin may normally stimulate actin polymerization in yeast (Yeh \& Haarer, 1996), its overproduction may serve to reduce the pool of actin available for polymerization (see Magdolen et al., 1993, for example).

As discussed by Yang et al. (1997), a better understanding of the connections between actin cytoskeletal function and bipolar budding will come with identification of the bipolar positional cue(s) and a determination of how such molecules achieve their polarized distribution. Our results (this study and Haarer et al., 1996), suggest that these cues achieve polarization through an actin-dependent, Myo2p-based and probably secretory pathway-based, delivery to the cell surface. Continued analysis of factors that affect cytoskeletal organization and cell polarization, particularly bipolar budding, will help establish the identity and activities of such positional determinants.

\section{ACKNOWLEDGEMENTS}

We would like to thank G. Fink, D. Drubin, K. Wertman, S. Lillie and S. Brown for generously providing strains and plasmids, D. Amberg for communicating unpublished results, and J. Chant for his major role in the development and performance of the bud-site selection screens. This work was supported a postdoctoral fellowship from the National Institutes of Health to T.F. and by grants from the NIH and the Searle Scholar/Chicago Community Trust to J. Chant (Harvard University) and from the National Science Foundation to B.H.

\section{REFERENCES}

Amberg, D. C., Basart, E. \& Botstein, D. (1995). Defining protein interactions with yeast actin in vivo. Nat Struct Biol 2, 28-35.

Amberg, D. C., Zahner, J. E., Mulholland, J. W., Pringle, J. R. \& Botstein, D. (1997). Aip3p/Bud6p, a yeast actin-interacting protein that is involved in morphogenesis and the selection of bipolar budding sites. Mol Biol Cell 8, 729-753.

Ayscough, K. R., Stryker, J., Pokala, N., Sanders, M., Crews, P. \& Drubin, D. G. (1997). High rates of actin filament turnover in budding yeast and roles for actin in establishment and maintenance of cell polarity revealed using the actin inhibitor latrunculin-A. J Cell Biol 137, 399-416.

Bauer, F., Urdaci, M., Aigle, M. \& Crouzet, M. (1993). Alteration of a yeast $\mathrm{SH} 3$ protein leads to conditional viability with defects in cytoskeletal and budding patterns. Mol Cell Biol 13, 5070-5084.

Bender, A. (1993). Genetic evidence for the roles of the bud-siteselection genes BUD5 and BUD2 in control of the Rsr1p (Bud1p) GTPase in yeast. Proc Natl Acad Sci U S A 90, 9926-9929.

Bender, A. \& Pringle, J. R. (1989). Multicopy suppression of the $c d c 24$ budding defect in yeast by CDC42 and three newly identified genes including the ras-related gene RSR1. Proc Natl Acad Sci U S A 86, 9976-9980.

Bobola, N., Jansen, R. P., Shin, T. H. \& Nasmyth, K. (1996). Asymmetric accumulation of Ash1p in postanaphase nuclei depends on a myosin and restricts yeast mating-type switching to mother cells. Cell 84, 699-709.

Cali, B. M., Doyle, T. C., Botstein, D. \& Fink, G. R. (1998). Multiple functions for actin during filamentous growth of Saccharomyces cerevisiae. Mol Biol Cell 9, 1873-1889.

Catlett, N. L. \& Weisman, L. S. (1998). The terminal tail region of a yeast myosin- $\mathrm{V}$ mediates its attachment to vacuole membranes and sites of polarized growth. Proc Natl Acad Sci USA 95, 14799-14804.

Chant, J. (1996). Generation of cell polarity in yeast. Curr Opin Cell Biol 8, 557-565.

Chant, J. \& Herskowitz, I. (1991). Genetic control of bud site selection in yeast by a set of gene products that constitute a morphogenetic pathway. Cell 65, 1203-1212.

Chant, J. \& Pringle, J. R. (1995). Patterns of bud-site selection in the yeast Saccharomyces cerevisiae. J Cell Biol 129, 751-765.

Chant, J., Corrado, K., Pringle, J. R. \& Herskowitz, I. (1991). Yeast $B U D 5$, encoding a putative GDP-GTP exchange factor, is necessary for bud site selection and interacts with bud formation gene BEM1. Cell 65, 1213-1224.

Chant, J., Mischke, M., Mitchell, E., Herskowitz, I. \& Pringle, J. R. 
(1995). Role of Bud3p in producing the axial budding pattern of yeast. J Cell Biol 129, 767-778.

Crouzet, M., Urdaci, M., Dulau, L. \& Aigle, M. (1991). Yeast mutant affected for viability upon nutrient starvation: characterization and cloning of the RVS161 gene. Yeast 7, 727-743.

Durrens, P., Revardel, E., Bonneu, M. \& Aigle, M. (1995). Evidence for a branched pathway in the polarized cell division of Saccharomyces cerevisiae. Curr Genet 27, 213-216.

Evangelista, M., Blundell, K., Longtine, M. S., Chow, C. J., Adames, N., Pringle, J. R., Peter, M. \& Boone, C. (1997). Bni1p, a yeast formin linking $\mathrm{Cdc} 42 \mathrm{p}$ and the actin cytoskeleton during polarized morphogenesis. Science 276, 118-122.

Finger, F. P. \& Novick, P. (1997). Sec3p is involved in secretion and morphogenesis in Saccharomyces cerevisiae. Mol Biol Cell 8, 647-662.

Finger, F. P., Hughes, T. E. \& Novick, P. (1998). Sec3p is a spatial landmark for polarized secretion in budding yeast. Cell 92, 559-571.

Gimeno, C. J., Ljungdahl, P. O., Styles, C. A. \& Fink, G. R. (1992). Unipolar cell divisions in the yeast $S$. cerevisiae lead to filamentous growth: regulation by starvation and RAS. Cell 68, 1077-1090.

Govindan, B., Bowser, R. \& Novick, P. (1995). The role of Myo2, a yeast class $\mathrm{V}$ myosin, in vesicular transport. J Cell Biol 128, 1055-1068.

Haarer, B. K., Lillie, S. H., Adams, A. E., Magdolen, V., Bandlow, W. \& Brown, S. S. (1990). Purification of profilin from Saccharomyces cerevisiae and analysis of profilin-deficient cells. J Cell Biol 110, 105-114.

Haarer, B. K., Petzold, A. S. \& Brown, S. S. (1993). Mutational analysis of yeast profilin. Mol Cell Biol 13, 7864-7873.

Haarer, B. K., Petzold, A., Lillie, S. H. \& Brown, S. S. (1994). Identification of MYO4, a second class $\mathrm{V}$ myosin gene in yeast. J Cell Sci 107, 1055-1064.

Haarer, B. K., Corbett, A., Kweon, Y., Petzold, A. S., Silver, P. \& Brown, S. S. (1996). SEC3 mutations are synthetically lethal with profilin mutations and cause defects in diploid-specific bud-site selection. Genetics 144, 495-510.

Halme, A., Michelitch, M., Mitchell, E. L. \& Chant, J. (1996). Bud10p directs axial cell polarization in budding yeast and resembles a transmembrane receptor. Curr Biol 6, 570-579.

Imamura, H., Tanaka, K., Hihara, T., Umikawa, M., Kamei, T., Takahashi, K., Sasaki, T. \& Takai, Y. (1997). Bni1p and Bnr1p: downstream targets of the Rho family small G-proteins which interact with profilin and regulate actin cytoskeleton in Saccharomyces cerevisiae. EMBO J 16, 2745-2755.

Jansen, R. P., Dowzer, C., Michaelis, C., Galova, M. \& Nasmyth, K. (1996). Mother cell-specific $H O$ expression in budding yeast depends on the unconventional myosin Myo4p and other cytoplasmic proteins. Cell 84, 687-697.

Jin, H. \& Amberg, D. C. (2000). The secretory pathway mediates localization of the cell polarity regulator Aip3p/Bud6p. Mol Biol Cell 11, 647-661.

Johnston, G. C., Prendergast, J. A. \& Singer, R. A. (1991). The Saccharomyces cerevisiae MYO2 gene encodes an essential myosin for vectorial transport of vesicles. J Cell Biol 113, 539-551.

Kron, S. J., Styles, C. A. \& Fink, G. R. (1994). Symmetric cell division in pseudohyphae of the yeast Saccharomyces cerevisiae. Mol Biol Cell 5, 1003-1022.

Lillie, S. H. \& Brown, S. S. (1992). Suppression of a myosin defect by a kinesin-related gene. Nature 356, 358-361.

Lillie, S. H. \& Brown, S. S. (1994). Immunofluorescence localization of the unconventional myosin, Myo2p, and the putative kinesin-related protein, Smy1p, to the same regions of polarized growth in Saccharomyces cerevisiae. J Cell Biol 125, 825-842.

Long, R. M., Singer, R. H., Meng, X., Gonzalez, I., Nasmyth, K. \& Jansen, R. P. (1997). Mating type switching in yeast controlled by asymmetric localization of ASH1 mRNA. Science 277, 383-387.

Lorenz, M. C. \& Heitman, J. (1997). Yeast pseudohyphal growth is regulated by $G P A 2$, a $G$ protein alpha homolog. EMBO $J \mathbf{1 6}$, 7008-7018.

Ma, H., Kunes, S., Schatz, P. J. \& Botstein, D. (1987). Plasmid construction by homologous recombination in yeast. Gene $\mathbf{5 8}$, 201-216.

Magdolen, V., Drubin, D. G., Mages, G. \& Bandlow, W. (1993). High levels of profilin suppress the lethality caused by overproduction of actin in yeast cells. FEBS Lett 316, 41-47.

Mosch, H. U. \& Fink, G. R. (1997). Dissection of filamentous growth by transposon mutagenesis in Saccharomyces cerevisiae. Genetics 145, 671-684.

Novick, P. \& Botstein, D. (1985). Phenotypic analysis of temperature-sensitive yeast actin mutants. Cell 40, 405-416.

Palmieri, S. J. \& Haarer, B. K. (1998). Polarity and division site specification in yeast. Curr Opin Microbiol 1, 678-686.

Park, H. O., Chant, J. \& Herskowitz, I. (1993). BUD2 encodes a GTPase-activating protein for Bud1/Rsr1 necessary for proper bud-site selection in yeast. Nature 365, 269-274.

Powers, S., Gonzales, E., Christensen, T., Cubert, J. \& Broek, D. (1991). Functional cloning of BUD5, a CDC25-related gene from $S$. cerevisiae that can suppress a dominant-negative RAS2 mutant. Cell 65, 1225-1231.

Pringle, J. R., Preston, R. A., Adams, A. E., Stearns, T., Drubin, D. G., Haarer, B. K. \& Jones, E. W. (1989). Fluorescence microscopy methods for yeast. Methods Cell Biol 31, 357-435.

Revardel, E., Bonneau, M., Durrens, P. \& Aigle, M. (1995). Characterization of a new gene family developing pleiotropic phenotypes upon mutation in Saccharomyces cerevisiae. Biochim Biophys Acta 1263, 261-265.

Roemer, T., Vallier, L. G. \& Snyder, M. (1996a). Selection of polarized growth sites in yeast. Trends Cell Biol 6, 434-441.

Roemer, T., Madden, K., Chang, J. \& Snyder, M. (1996b). Selection of axial growth sites in yeast requires Axl2p, a novel plasma membrane glycoprotein. Genes Dev 10, 777-793.

Ruggieri, R., Bender, A., Matsui, Y., Powers, S., Takai, Y., Pringle, J. R. \& Matsumoto, K. (1992). RSR1, a ras-like gene homologous to Krev-1 (smg21A/rap1A): role in the development of cell polarity and interactions with the Ras pathway in Saccharomyces cerevisiae. Mol Cell Biol 12, 758-766.

Sambrook, J., Fritsch, E. F. \& Maniatis, T. (1989). Molecular Cloning: a Laboratory Manual, 2nd edn. Cold Spring Harbor, NY: Cold Spring Harbor Laboratory.

Sanders, S. L. \& Herskowitz, I. (1996). The BUD4 protein of yeast, required for axial budding, is localized to the mother/BUD neck in a cell cycle-dependent manner. J Cell Biol 134, 413-427.

Schott, D., Ho, J., Pruyne, D. \& Bretscher, A. (1999). The COOHterminal domain of Myo2p, a yeast myosin V, has a direct role in secretory vesicle targeting. J Cell Biol 147, 791-808.

Sherman, F., Fink, G. R. \& Hicks, J. B. (1986). Methods in Yeast Genetics. Cold Spring Harbor, NY: Cold Spring Harbor Laboratory.

Snyder, M. (1989). The SPA2 protein of yeast localizes to sites of cell growth. J Cell Biol 108, 1419-1429. 
Sohn, R. H. \& Goldschmidt-Clermont, P. J. (1994). Profilin: at the crossroads of signal transduction and the actin cytoskeleton. Bioessays 16, 465-472.

Takizawa, P. A., Sil, A., Swedlow, J. R., Herskowitz, I. \& Vale, R. D. (1997). Actin-dependent localization of an RNA encoding a cell-fate determinant in yeast. Nature 389, 90-93.

Ward, M. P., Gimeno, C. J., Fink, G. R. \& Garrett, S. (1995). SOK2 may regulate cyclic AMP-dependent protein kinase-stimulated growth and pseudohyphal development by repressing transcription. Mol Cell Biol 15, 6854-6863.

Yang, S., Ayscough, K. R. \& Drubin, D. G. (1997). A role for the actin cytoskeleton of Saccharomyces cerevisiae in bipolar bud-site selection. J Cell Biol 136, 111-123.

Yeh, J. \& Haarer, B. K. (1996). Profilin is required for the normal timing of actin polymerization in response to thermal stress. FEBS Lett 398, 303-307.

Zahner, J. E., Harkins, H. A. \& Pringle, J. R. (1996). Genetic analysis of the bipolar pattern of bud site selection in the yeast Saccharomyces cerevisiae. Mol Cell Biol 16, 1857-1870.

Received 1 April 2000; revised 24 August 2000; accepted 7 August 2000. 